\section{Vol. 32, Issue 5, May 2009}

Obituary

234 'Psychosocial Oncology Does not Give Less Regard to the Body but More to the Soul' - Obituary Prof. Dr. med. Dipl.-Soz. Reinhold Schwarz (1946 - 2008) Kuhnt, S.; Lehmann, A. (Leipzig)

\section{Editorial}

235 Where Do We Place PankoMab in the Reagents Used to Study the MUC1 Superfamily? Li, J.; Sullivan, C.A.W.; Harris, L.N. (New Haven, CT)

Original Articles

238 Evaluation of a Novel Anti-Mucin 1 (MUC1) Antibody (PankoMab) as a Potential Diagnostic Tool in Human Ductal Breast Cancer; Comparison with Two Established Antibodies Dian, D.; Janni, W.; Kuhn, C.; Mayr, D. (München); Karsten, U. (Berlin); Mylonas, I.; Friese, K.; Jeschke, U. (München)

246 CD4+CD25+ Regulatory T Cells in Patients with Advanced Gastrointestinal Cancer Treated with Chemotherapy Xu, H.; Mao, Y.; Dai,Y.; Wang, Q.; Zhang, X (Suzhou)

254 CT-Guided Percutaneous Core Needle Biopsy in Oncology Outpatients: Sensitivity, Specificity, Complications Steil, S.; Zerwas, S.; Moos, G.; Bittinger, F.; Hansen, T. Mergenthaler, U.; Weide, R. (Koblenz)

260 Is Prostate Cancer Screening Justified in the Bedouin Population?

Abdallah, M.; Lazarev, I.; Ariad, S.; Mermershtain, W. (Beer Sheva)

264 Single Tissue Samples from Head and Neck Squamous Cell Carcinomas are Representative Regarding the Entire Tumor's Chemosensitivity to Cisplatin and Docetaxel

Wichmann, G.; Horn, I.-S.; Boehm, A.; Mozet, C.; Tschöp, K. (Leipzig); Dollner, R. (Oslo); Dietz, A. (Leipzig)

\section{Band 32, Heft 5, Mai 2009}

Nachruf

234 «Die Psychosoziale Onkologie schenkt nicht dem Körper weniger, sondern der Seele mehr Aufmerksamkeit» - Nachruf Prof. Dr. med. Dipl.-Soz. Reinhold Schwarz (1946 - 2008) Kuhnt, S.; Lehmann, A. (Leipzig)

Editorial

235 Welchen Stellenwert hat PankoMab unter den Antikörpern, mit denen die MUC1-Familie untersucht wird? Li, J.; Sullivan, C.A.W.; Harris, L.N. (New Haven, CT)

Originalarbeiten

238 Evaluierung eines neuen Anti-Mucin 1 (MUC1)-Antikörpers (PankoMab) als ein potenzielles diagnostisches Mittel beim duktalen Mammakarzinom; und dessen Vergleich mit zwei etablierten Antikörpern Dian, D.; Janni, W.; Kuhn, C.; Mayr, D. (München); Karsten, U. (Berlin); Mylonas, I.; Friese, K.; Jeschke, U. (München)

246 CD4+CD25+ regulatorische T-Zellen bei Patienten mit fortgeschrittenen gastrointestinalen Tumoren unter Chemotherapie Xu, H.; Mao, Y.; Dai,Y.; Wang, Q.; Zhang, X (Suzhou)

254 Die CT-gesteuerte, perkutane Stanzbiopsie bei ambulanten onkologischen Patienten: Sensitivität, Spezifität, Komplikationen Steil, S.; Zerwas, S.; Moos, G.; Bittinger, F.; Hansen, T.; Mergenthaler, U.; Weide, R. (Koblenz)

260 Ist Prostatakrebs-Screening in Beduinenpopulationen sinnvoll?

Abdallah, M.; Lazarev, I.; Ariad, S.; Mermershtain, W. (Beer Sheva)

264 Einzelne Biopsate von Plattenepithelkarzinomen von Kopf und Hals sind repräsentativ für die Chemosensitivität des gesamten Tumors gegenüber Cisplatin und Docetaxel

Wichmann, G.; Horn, I.-S.; Boehm, A.; Mozet, C.; Tschöp, K. (Leipzig); Dollner, R. (Oslo); Dietz, A. (Leipzig)

\section{KARGER}

Fax +497614520714

Information@Karger.de www.karger.com (c) 2009 S. Karger GmbH, Freiburg 


\section{Vol. 32, Issue 5, May 2009}

Clinical Cases

274 Video-Assisted Thoracoscopic Surgery for Recurrent Ovarian Cancer with a Metastatic Mediastinal Mass Ki, K.-D.; Lee, J.-M.; Tong, S.-Y.; Lee, S.-K.; Kim, D.-H.; Kwak, Y.-T.; Lim, S.-J. (Seoul)

277 Purpura as the Initial Presentation for Small Cell Lung Cancer

Zoppoli, G.; Balleari, E.; Bruzzone, A.; Calvia, A.; Mastracci, L.; Ghio, R. (Genoa)

280 Symptomatic Hyponatraemia Caused by Cylophosphamide

Berger, A.; Bellos, F.; Siegmund, A.; Eisenbach, C.; Lordick, F. (Heidelberg)

283 Delayed Oxaliplatin-Induced Sensorimotor Polyneuropathy

Nardone, R. (Meran/Salzburg); Buratti, T. (Meran);

Golaszewski, S. (Salzburg); Bratti, A.; Caleri, F.; Tezzon, F. (Meran); Ladurner, G. (Salzburg); Mitterer, M. (Meran)

\section{Review Article}

286 Limitation of Surgical Radicality in Rectal Cancer Responding to Neoadjuvant Therapy

Troja, A.; Hempen, H.-G.; Raab, H.-R. (Oldenburg)

292 PharmaNews / PharmaTicker

295 Meetings and Conferences

299 Guidelines for Authors

237 Imprint

Forthcoming papers are listed on page 300.

\section{Band 32, Heft 5, Mai 2009}

Kasuistiken

274 Die videounterstuitzte thorakoskopische Chirurgie (VATS) bei Ovarialkarzinom-Rückfallpatienten mit mediastinalen Metastasen

Ki, K.-D.; Lee, J.-M.; Tong, S.-Y.; Lee, S.-K.; Kim, D.-H.; Kwak, Y.-T.; Lim, S.-J. (Seoul)

277 Purpura als initiale Erscheinung von kleinzelligem Bronchialkarzinom

Zoppoli, G.; Balleari, E.; Bruzzone, A.; Calvia, A.; Mastracci, L.; Ghio, R. (Genoa)

280 Symptomatische Hyponaträmie bei Behandlung mit Cyclophosphamid

Berger, A.; Bellos, F.; Siegmund, A.; Eisenbach, C.;

Lordick, F. (Heidelberg)

283 Verzögerte oxaliplatininduzierte sensorimotorische Polyneuropathie

Nardone, R. (Meran/Salzburg); Buratti, T. (Meran);

Golaszewski, S. (Salzburg); Bratti, A.; Caleri, F.; Tezzon, F. (Meran);

Ladurner, G. (Salzburg); Mitterer, M. (Meran)

Übersichtsarbeit

286 Einschränkung der chirurgischen Radikalität beim Rektumkarzinom nach Remission unter neoadjuvanter Therapie

Troja, A.; Hempen, H.-G.; Raab, H.-R. (Oldenburg)

292 PharmaNews / PharmaTicker

295 Tagungen und Kongresse

299 Hinweise für Autoren

237 Impressum

Einen Ausblick auf den Inhalt der kommenden Hefte finden Sie auf Seite 300 .

\section{KARGER}

Fax +49761 4520714

Information@Karger.de

www.karger.com
(C) 2009 S. Karger GmbH, Freiburg 